\title{
Critical Imperative for the Reform of British Interpretation of Fetal Heart Rate Decelerations: Analysis of FIGO and NICE Guidelines, Post-Truth Foundations, Cognitive Fallacies, Myths and Occam's Razor
}

\author{
Shashikant L. Sholapurkar
}

\begin{abstract}
Cardiotocography (CTG) has disappointingly failed to show good predictability for fetal acidemia or neonatal outcomes in several large studies. A complete rethink of CTG interpretation will not be out of place. Fetal heart rate (FHR) decelerations are the most common deviations, benign as well as manifestation of impending fetal hypoxemia/acidemia, much more commonly than FHR baseline or variability. Their specific nomenclature is important (center-stage) because it provides the basic concepts and framework on which the complex "pattern recognition" of CTG interpretation by clinicians depends. Unfortunately, the discrimination of FHR decelerations seems to be muddled since the British obstetrics adopted the concept of vast majority of FHR decelerations being "variable" (cord-compression). With proliferation of confusing waveform criteria, "atypical variables" became the commonest cause of suspicious/pathological CTG. However, National Institute for Health and Care Excellence (NICE) (2014) had to disband the "typical" and "atypical" terminology because of flawed classifying criteria. This analytical review makes a strong case that there are major and fundamental framing and confirmation fallacies (not just biases) in interpretation of FHR decelerations by NICE (2014) and International Federation of Gynecology and Obstetrics (FIGO) (2015), probably the biggest in modern medicine. This "post-truth" approach is incompatible with scientific practice. Moreover, it amounts to setting oneself for failure. The inertia to change could be best described as "backfire effect". There is abundant evidence that head-compression (and other non-hypoxic mediators) causes rapid rather than shallow/gradual decelerations. Currently, the vast majority of decelerations are attributed to unproven cord compression underpinned by flawed disproven pathophysiological hypotheses. Their further discrimination based on abstract, random, trial and error criteria remains unresolved suggesting a false premise to begin with. This is not surprising considering that the commonest pathophysiology of intrapartum hypoxemia is contraction-induced reduction in uteroplacental perfusion (sometimes already compromised)
\end{abstract}

Manuscript accepted for publication January 23, 2017

Department of Obstetrics and Gynaecology, Royal United Hospital Bath NHS Foundation Trust, Combe Park, Bath, BA1 3NG, UK.

Email: s.sholapurkar@nhs.net

doi: https://doi.org/10.14740/jocmr2877e and not cord compression at all. This distorted categorization causes confusion, false-alarm fatigue and difficulty in focusing on real pathological decelerations making CTG interpretation dysfunctional ultimately compromising patient safety. Obstetricians/midwives should demand reverting to the previous more scientific British categorization of decelerations based solely on time relationship to contractions as advocated by the pioneers like Hon and Caldeyro-Barcia, rather than accepting the current "post-truth" scenario.

Keywords: Cardiotocography; Fetal heart rate decelerations; Intrapartum fetal monitoring; Confirmation bias; Framing bias; FIGO CTG guidelines; NICE CTG guidelines; Intermittent auscultation

\section{Introduction}

Cardiotocography (CTG) interpretation remains controversial and seems to have become even more confusing since the turn of the century. National professional bodies have repeatedly changed their guidelines in the last two decades. Atypical variable fetal heart rate (FHR) decelerations had become the commonest cause of suspicious and pathological CTGs in UK after 2007. However, in 2014, the National Institute for Health and Care Excellence (NICE) made a major correction by disbanding the subcategorization of variable FHR decelerations into typical and atypical which raises doubts about the science behind its previous stance [1,2]. Currently, the British obstetricians are divided on whether to follow NICE (2014) or the International Federation of Gynecology and Obstetrics (FIGO, $2015)$ guidelines or some other locally modified "convenient" version $[1,3]$. In the USA, it was reported that the three-tier system of American Congress of Obstetricians and Gynecologists $(\mathrm{ACOG})[4,5]$ was unhelpful in clinical practice [6-8]. Different conflicting proposals and guidelines have led to so much confusion and uncertainty that the CTG interpretation has been claimed to be a "junk science" (completely lacking evidence) to be barred from court rooms based on Daubert principle [9]. In UK, the recently completed "INFANT" [10] randomised controlled trial (RCT) of CTG (as currently practiced) and additional intelligent software system support has given very disappointing results (data awaiting publication). A recent robust American study reported that at the most $50 \%$ of 
acidemic babies could have been detected by a retrospective CTG analysis by experts in a highly artificial environment in which attention to detail was unhampered by the need to provide actual care to multiple patients [11]. But that study also concluded that under practical realistic conditions, this "expert CTG protocol" could be effective in expediting delivery of somewhat lesser number of (probably closer to $30 \%$ ) acidemic babies [11]. Thus, a fundamental rethink seems necessary to resuscitate the practice of CTG in Europe and America. A prominent paper described FHR decelerations to be "center-stage" in CTG interpretation [12], because they are the commonest aberrations on CTG with maximum influence on three-tier classification. They are singularly the most characteristic manifestation of impending fetal hypoxemia, more than FHR baseline or variability [13]. An editor's choice paper reported that the "peculiar nature" of American categorization of FHR decelerations (adopted in UK after 2007) may have been responsible for the loss of meaning [7]. Thus, a meaningful analysis and understanding of FHR decelerations and the underlying pathophysiology would underpin the functionality of three-tier systems [13]. This raises a legitimate question as to why the longstanding traditional British practice of classification of FHR decelerations (based primarily on timing) [14-17] before 2007 was abandoned. The categorization systems of FHR decelerations are theoretical constructs. Any framing/anchoring and confirmation biases in categorization of FHR decelerations should be corrected even simply for a basic scientific approach especially if these amount to fundamental and material flaws/fallacies (not just logical biases). A mistaken categorization of FHR decelerations can be shown to lead to further (forced) errors and inaccuracies. This review will provide evidence whether the current NICE (2014) and FIGO (2015) definitions of FHR decelerations [1,3] may be unscientific and unsound. It will encourage a debate about restoring the previous British categorization of FHR decelerations purely based on time relationship to contractions. Other parameters like FHR baseline, variability or accelerations are not the subject of this paper but of course have always formed a part of overall picture. Notably, the recent guidelines have not made any significant changes to these FHR parameters which remain relatively uncontroversial in contrast to FHR decelerations.

\section{Critical Thinking: Framing, Confirmation and Other Biases or Fallacies}

Cognition is what makes us truly human (Rene Decartes "You think, therefore you are!"). Our minds can amaze but also betray us [18]. We make sense of the world by forming concepts which simplify our thinking in a fundamental way [18]. Concepts allow us to make quick, instinctive but insightful decisions especially when interpretation of complex and multiple features are involved (e.g. CTG patterns). We organize our concepts by defining prototypes - mental images or pinnacle examples of certain things. Although concepts and prototypes can speed up our thinking, they can also "box in" our thinking and could lead to biases and fallacies. Even worse would be being "boxed in" by fallacious concepts! Hence, we must actively keep our minds open for evolving concepts and intruding biases [18].

The renowned British philosopher and a Nobel laureate mathematician Bertrand Russell pointed out that science is a small area of known knowledge and moreover, there are things we thought we knew but do not know. Most of the CTG interpretation remains unproven despite the advent of evidencebased medicine (EBM). "Critical analysis" (a branch of philosophy) is the way to make sense of things not yet proven by science. Critical thinking (not synonymous with criticism) is making sure we have good reasons for our beliefs or hypotheses. Sometimes myths are more powerful or attractive than facts. Failure to think critically comes with serious consequences. A key part of critical thinking is to evaluate if arguments (premises and resultant conclusions) are good or bad. This requires avoidance of many common inherent cognitive biases. "Confirmation bias" occurs when we selectively focus upon evidence that supports our beliefs, while ignoring more comprehensive evidence that disproves these ideas [18-20]. It is closely related to "framing/anchoring bias" which is the tendency to create coherent initial picture without examining all available information $[19,20]$. Our cognition is affected by how things are presented, i.e. "framing" affects our decisions and judgements. A type of framing bias is used by advertisers or salespeople, e.g. advertising a price discount or offers such as "three for the price of two". The decision to buy or not can be influenced by this "biased framing" but neither decision would necessarily be wrong. However, if the "framing" is completely fallacious (e.g. fake goods are offered as genuine), then a decision to buy would almost invariably be wrong. Hence, fallacious framing should not be accepted. Confirmation bias (belief perseverance) is common or even ubiquitous but quite hazardous and very difficult to avoid [18, 19]. A good way of minimizing confirmation bias is to actively seek contradictory theories or arguments and test or address them [13]. Another well-described bias is "genetic bias" where we accept or reject an idea because of its origin or history. Genetic bias is not always wrong or can even be useful because it allows us to rely on expert opinion/committees and reputed scientific publications which therefore have a special responsibility. Hence, they should be able to support their ideas with critical reasoning when scientific proof is lacking. All these biases can lead to tunnel vision and to the undesirable phenomenon of "group polarization" (a better description than "group think") and can develop into major fallacies. With group polarization it is well known that a voice trying to point out that the emperor has no clothes is ignored or worse oppressed.

\section{Pioneering Work of Hon and Low-Lying Fruit}

Edward Hon and Caldeyro-Barcia were the pioneers who intuitively recognized three main categories of FHR decelerations well before the advent of EBM [21,22]. These pioneers had quite quickly observed that decelerations with "early" timing were benign and those with "late" timing were likely to be associated with fetal hypoxemia. This timing-based categoriza- 


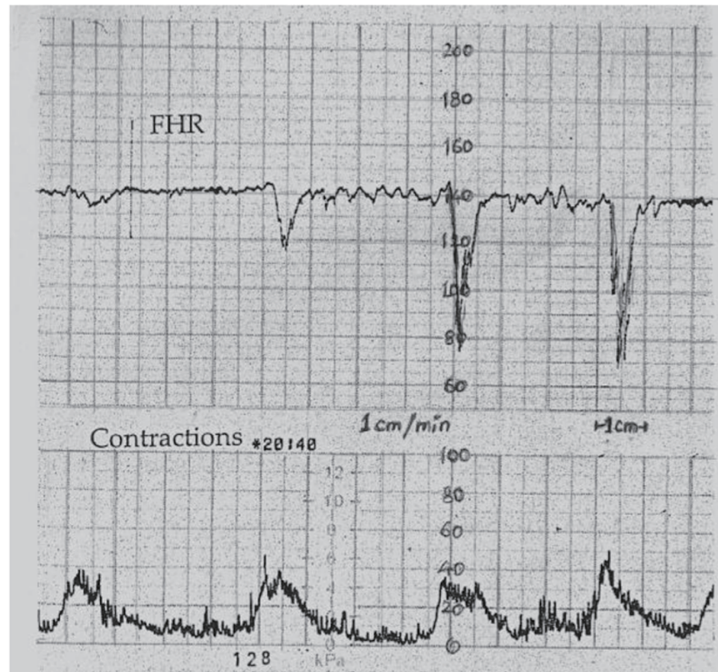

Early decelerations (Type 1, or ' $\mathrm{V}$ ' dip). The . nadir of the deceleration coincides with the peak of uterine contraction and there is a rapid return to the baseline of approximately 140 beats/minute

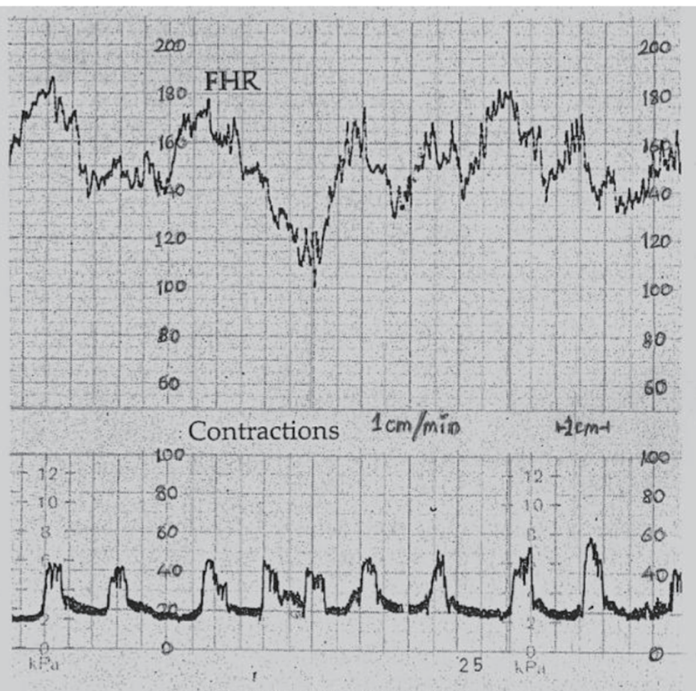

Late decelerations (Type 2, or ' $\mathrm{U}^{\prime}$ dip). There is a baseline tachycardia. Decelerations are of longer duration than with Type 1 dips and continue after the contraction has passed

Figure 1. Early and late decelerations in traditional British practice (Reproduced with permission from "Principles of Obstetrics" by Bryan Hibbard, 1988) [17]. Note "rapid rather than gradual descent" of early as well as some of the late FHR decelerations. Illustrations by other British authorities before 2007 were also very similar [14-16]. Many old as well as recent illustrations [25-27] show that late decelerations on British CTG can look more like "V" than "U" especially when they are deep (more serious). Hence there is a danger of miscategorising late decelerations if "gradual" shape is included in the British definition of late decelerations.

tion represented the "low-lying fruit" which can be picked up by simple heuristic methodology (observation, intuitive judgement, profiling, disambiguation, etc.) and hence generally have a high strength of correlation [23]. Advanced research methodologies of EBM are gold-standard but at times have increasingly become a science of marginal gains [23]. This rings particularly true of inconclusive research on CTG interpretation in the last 50 years which is mainly because of the wide variations in methodology, definitions, intrinsic complexities of the subject and fundamental fallacies which may have crept in [13]. Hence, it is particularly important to preserve the early gains of the "low-lying fruit". Methodologies of EBM will not compensate for fundamental major framing fallacies. All international categorizations of FHR decelerations even today claim to be based on the description by Hon who defined early, late and variable decelerations (Caldeyro-Bracia - type I, II and III) primarily based on their time relationship to the contractions $[21,22]$. As a secondary observation, Hon proposed that early, late and variable decelerations are likely to be due to head-compression, hypoxemia and cord compression, respectively [21]. Etiology of decelerations will remain presumptive and is likely to be multifactorial with one of the causes predominant [24]. Hon made a passing "hypothesis" that the shape of early decelerations should mimic the curve of rise of intrauterine pressure but quickly ruled it out by stating that this cannot be the case in actual practice because of the scale selected for display on the CTG [21]. The shape (gradual/rapid) and size of waveforms is not absolute but dependent on scale of display which is a matter of convenient choice or conven- tion [24]. It is a misconception to infer from Hon's description (and some illustrations with altered scale) that early decelerations should be shallow/gradual by selectively choosing some of his very rare, preliminary and tentative narratives especially when he was referring to CTG traces with a faster recording speed $(3 \mathrm{~cm} / \mathrm{min})$ anyway.

\section{Misguided Discontinuation of the Traditional British Categorization of FHR Decelerations}

The term "traditional" British categorization of FHR decelerations here refers to the practice between 1970 and 2007 which can be inferred from the British mainstream textbooks [14-17]. It was very much based on the specific definitions by Hon [21] and did not require early decelerations to be shallow/gradual (Figs. 1 and 2) [14-17, 25-27]. A survey of senior British obstetricians and midwives revealed that before 2007, "early" decelerations constituted $50-70 \%$ of all decelerations [24, 28]. The three-tier system of the Royal College of Obstetricians and Gynaecologists (RCOG) [29] seemed to work well with the traditional British categorization of FHR decelerations. However, the subsequent generation of opinion leaders at the time of NICE (2007) guidelines [30] enforced a major change by stating that the vast majority of decelerations are due to cord compression and hence must be called variable (in line with American practice). This was achieved by enforcing a mythical concept that the early and late decelerations must be truly uniform (same in size and shape) $[25,30]$ and gradual. 


\section{PERIODIC VARIATIONS}

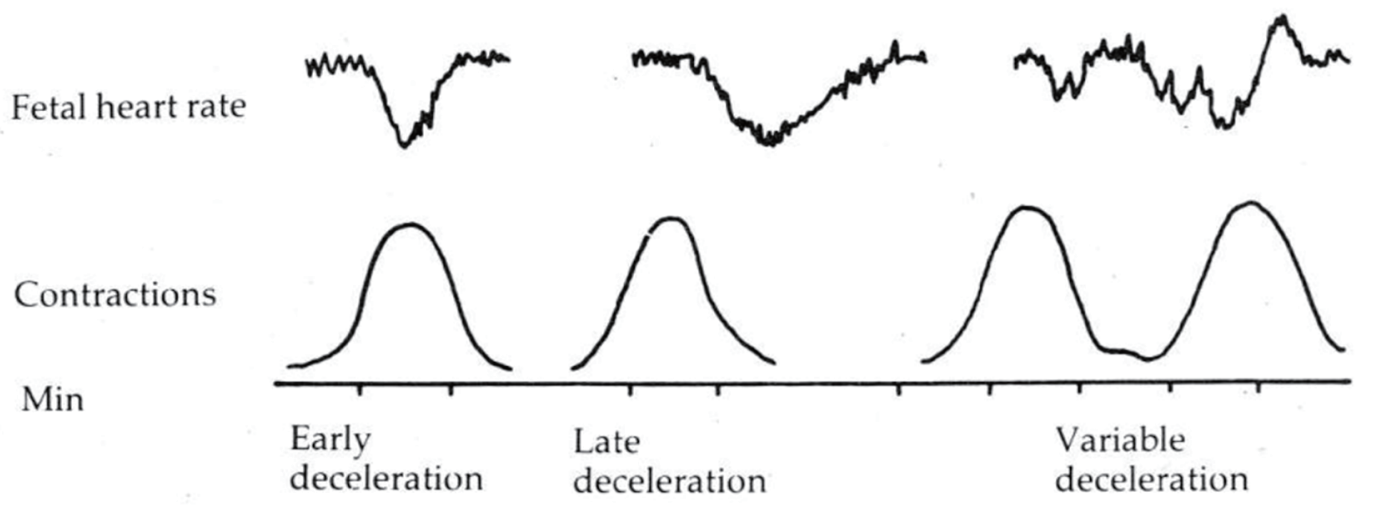

\section{Caldeyro \\ Barcia}

Hon

Principal

cause
Type 1

Type 2

Udip

Uteroplacental

insufficiency

(UPI)
Umbilical cord

compression

(ml)

Figure 2. Diagrammatic representation of early, late and variable decelerations as practiced in British Obstetrics before 2007 (Reproduced with permission from "Principles of Obstetrics" by Bryan Hibbard, 1988) [17]. Etiology is not a primary defining feature. Late decelerations are described as "U"-shaped only in contrast with "V"-shaped early decelerations.

Consequently, with near extinction of early and late decelerations, the midwives who are the front-line workers reported that CTG interpretation had become dysfunctional because of completely new-found difficulties in pattern recognition, thus affecting their performance and confidence [2, 28]. On the other hand, many senior obstetricians have simply continued to interpret CTGs based on their past experience and conceptual understanding of the traditional British categorization of decelerations using the flexibility and discretion they can exercise [14-17, 24, 28].

\section{American Categorization of FHR Decelerations: Framing and Confirmation Fallacies}

American categorization of decelerations $[4,5]$ seems to have materially deviated from and in fact contradictory to that of Hon [21] without being explicit, open or transparent about it. Because the animal experiments demonstrated that artificial cord occlusion resulted in rapid drop in FHR, all decelerations with rapid descent (arbitrarily baseline to trough $<30 \mathrm{~s}$ ) were assumed to be because of cord compression and hence called "variable" $[4,5]$. In contrast, the head-compression was postulated to cause shallow or gradual decelerations almost as an ideological belief. It could be debated if the findings from animal experiments, often involving "complete" cord occlusion for $30-60 \mathrm{~s}$, could be extrapolated to relatively common FHR decelerations in human labor. The current FHR deceleration categorization has become an "etiological classification" linked to rate of descent. An elaborate cord-compression hypothesis [25-27, 31, 32] was designed to explain the common rapid decelerations during contractions (Fig. 3) [12, 13, $25-27,31,32,33]$, but has been shown to be seriously flawed [13]. The entire concept of gradual/rapid decelerations signifying head/cord-compression respectively has been shown to be riddled with framing/anchoring and confirmation fallacies incompatible with scientific wisdom [13]. A token example is a description by an American expert below [34].

"Early decelerations are shallow, symmetric, uniform with onset and return that are gradual resulting in a "U"-shaped decelerations. These are thought to be caused by compression of fetal head by uterine cervix as it over-rides anterior frontanelle of the cranium and occurs between 4 and $6 \mathrm{~cm}$ of cervical dilatation. More non-specific head-compression can result in decelerations indistinguishable from variable decelerations, and these latter decelerations have often been called early decelerations, but are by definition not so" [34].

The above detailed specification of waveform shapes (some of it already defunct) creates a facade of alluring and authoritative certainty. This description shows that rather than questioning the framing/anchoring fallacy in the definition (shallow/gradual) of early decelerations, it has been further supported by a "confirmation fallacy/bias" that all rapid decelerations must be called "variable" by definition even if many of them are due to head-compression. Head-compression is very common in first as well as second stage of labor as we know molding and caput develops throughout the active labor. Hence it does not seem rational or likely that hardly any "early" decelerations will be found or they would be mostly limited between 4 and $6 \mathrm{~cm}$ cervical dilatation. 


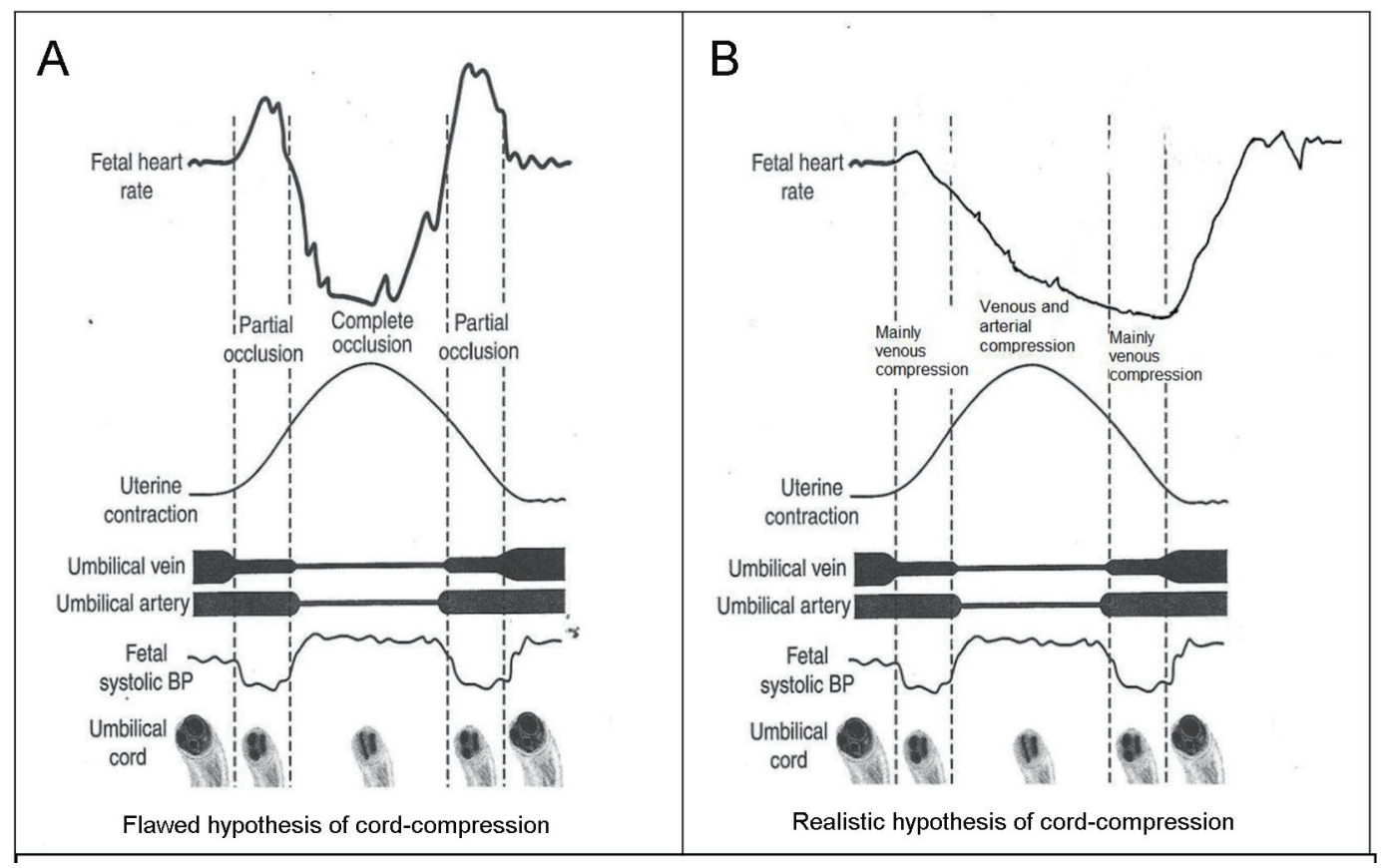

Figure 3. (A) Popular but flawed hypothesis of cord-compression and variable deceleration [25-27, 31, 32]. CTG speed $3 \mathrm{~cm} / \mathrm{min}$. Thankfully reproduced from J Clin Med Res. 2015;7:672-80 [13]. This hypothesis has major fallacies. Complete cord-compression has been postulated for these most common decelerations. FHR recovery commencing at the height of contraction (where umbilical arterial and venous occlusion is unrelieved) cannot be explained. Instead the deceleration depicted seems consistent with "non-hypoxic" vagal reflex (e.g. head-compression). (B) Diagrammatic illustration of how "cord-compression" deceleration would actually look like (CTG speed $3 \mathrm{~cm} / \mathrm{min}$ ). Both baro and chemoreceptor mechanisms come into play as shown by many animal studies [12, 33]. The shape will look more rapid (V shaped) on British CTG (speed $1 \mathrm{~cm} / \mathrm{min}$ ). The nadir is reached well after the peak of contraction and recovery would start when umbilical vein compression (i.e. hypoxemia) is being relieved. This is clearly borne out in the Figure 5, where an example of FHR deceleration with known cord compression is given [12].

\section{Critical Analysis of FIGO (2015) Definitions of Decelerations}

Expert committees have stressed that it is of vital importance that the definitions of FHR parameters should be specific, standardized and unambiguous [5]. This would be particularly important about FHR decelerations which are the most important, common and complex waveforms requiring conceptualization and pattern recognition during CTG interpretation. Recently FIGO (2015) published new guidelines on intrapartum fetal monitoring [3]. The FIGO definitions of FHR decelerations (similar to current British practice and NICE guidelines) need to be critically examined by the obstetricians and midwives for their validity and any disambiguation/reform required.

\section{FIGO (2015) definition of early decelerations}

Decelerations that are shallow, short-lasting, with normal variability within the deceleration and are coincident with contractions. They are believed to be caused by fetal head-compression [35] and do not indicate fetal hypoxemia/acidosis [3].

Despite retaining the nomenclature "early" by Hon [21] clearly denoting/signifying "timing", it is noteworthy that the morphological feature (shallow) seems to have become the primary defining criterion by FIGO (and NICE). However, the decelerations that look gradual on American CTG (recording speed $3 \mathrm{~cm} / \mathrm{min}$ ) will appear rapid on a British CTG (recording speed $1 \mathrm{~cm} / \mathrm{min}$ ). Let's consider what actually makes the decelerations appear gradual versus rapid on a British CTG record. Figure 4 shows the actual physical reality that to appear gradual/shallow, the decelerations will need width to depth ratio of about 2:1 or more. Figure 4 further demonstrates that to "appear" shallow/gradual, a deceleration with smallest depth of $15 \mathrm{bpm}$ will need duration of about $90 \mathrm{~s}$ or more, and a medium size deceleration (depth $30 \mathrm{bpm}$ ) will need duration of about $3 \mathrm{~min}$ or more. Hence, it seems physically impossible to have "short-lasting gradual decelerations" on a British CTG (depth more than $15 \mathrm{bpm}$ in the presence of normal variability). The term "shallow" is a further source of confusion because it could be misinterpreted as less than $15 \mathrm{bpm}$ in depth. But, there has never been a concept of early decelerations of less than $15 \mathrm{bpm}$ nor is it rational (unlike late decelerations on the background of reduced baseline variability) $[1,4,5,21$, $22,29,30,27]$. Thus, "short-lasting" and "shallow/gradual" decelerations are a paradox and a contradiction in the FIGO definition itself (Fig. 4), which makes the definition void. Similar contradictions also apply to American CTG even after the adoption of completely arbitrary $30 \mathrm{~s}$ cut-off for descent time $[13,33]$. Equally importantly, there is no evidence for the concept that head-compression causes "shallow" decelerations on 


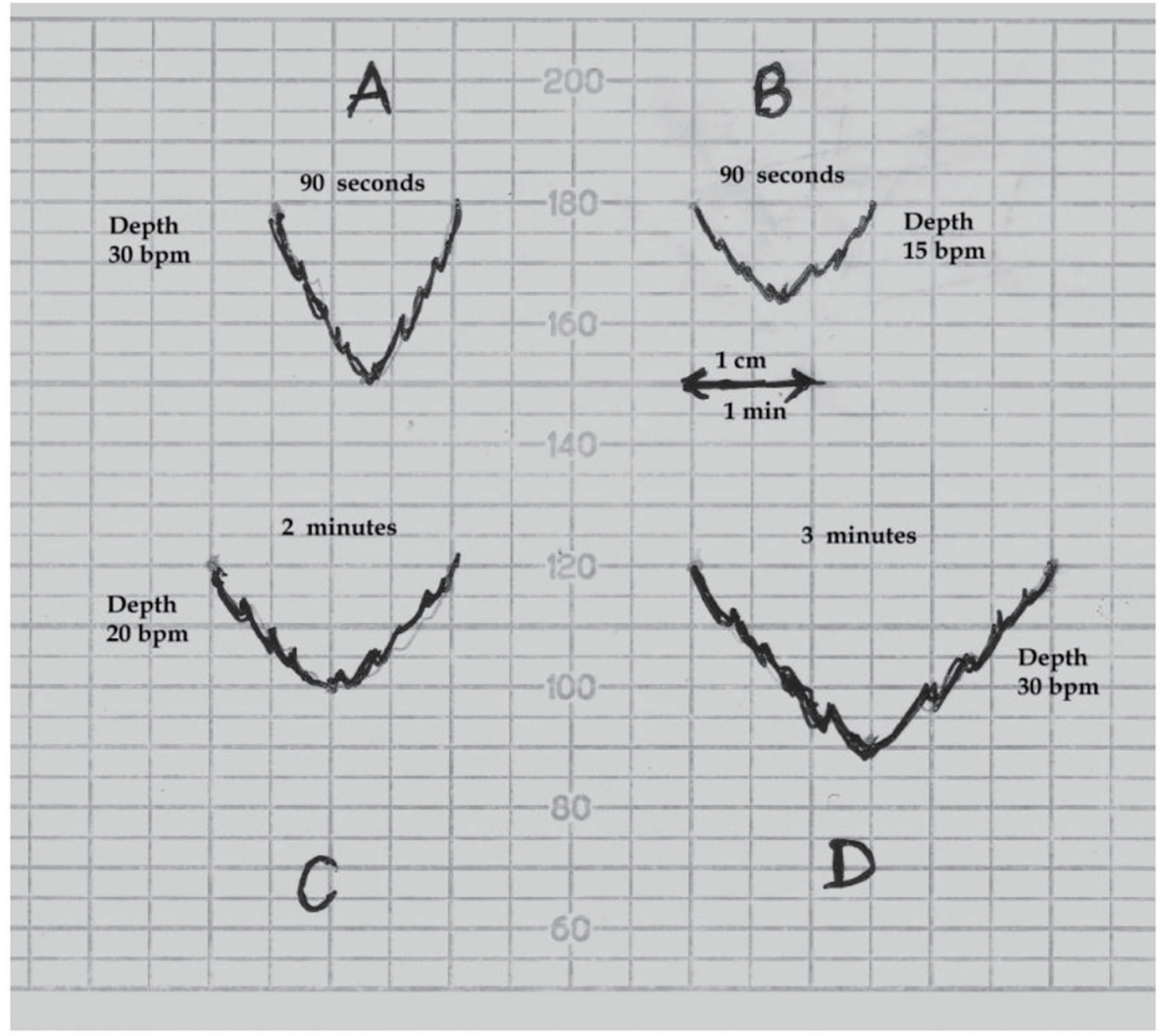

Figure 4. Schematic drawing of FHR decelerations on the British CTG paper (recording speed $1 \mathrm{~cm} / \mathrm{min}$ ). bpm: beats per minute. (A) This deceleration with W/D (width to depth) ratio of 1:1 or less will appear "rapid". (B) A deceleration will need W/D ratio of about 2:1 or more to appear "shallow/gradual". Thus a smallest deceleration of depth $15 \mathrm{bpm}$ will need to be about $90 \mathrm{~s}$ or more in duration to appear "shallow". (C, D) Decelerations of depth 20 and $30 \mathrm{bpm}$ will need to be around 2 and 3 min or more in duration respectively to appear "shallow". Thus "shallow/gradual and short lasting" decelerations coincident with contractions are physically not possible on British CTG.

British or American CTG [13]. This misconception and flawed definition may explain why early decelerations are hardly ever found despite the common occurrence of head-compression in labor. Most importantly, the sole reference given by FIGO (2015) for this peculiar definition [3] is that of eminent American expert Prof. J. Parer [35]. But notwithstanding, Prof. Parer in a subsequent detailed seminal paper repeatedly asserted that head-compression actually causes "rapid" decelerations indistinguishable from the so-called "variable" (rapid) decelerations [36]. He also quoted other experimental studies in human labor including those by Hon where application of pressure to the fetal head produced "rapid" decelerations [37, 38]. The group of Caldeyro-Barcia also demonstrated type I "rapid" decelerations on application of pressure to fetal head to mimic that during labor contractions [22]. Hence, FIGO and other similar definitions of "early" decelerations may misinform the young obstetricians and midwives who then get hardwired to it. Respected British philosopher John Gray has quoted, "When one is inside a myth, it feels like a fact; and myths can be more powerful (or attractive) than facts." He further added that some myths are abandoned only when shown to be completely ridiculous or power-structures sustaining those myths dwindle. Myths are incompatible with scientific practice. Should obstetricians and midwives simply accept this post-factual situation comprising of very significant framing/anchoring fallacies and contradictions which make benign "early" decelerations nearly extinct and consequently distorting the CTG interpretation? Post-truth approach will invariably compromise science. Not surprisingly, FIGO (2015) has been unable to provide any illustration of "shallow/gradual short-lasting" early decelerations [3]. Moreover, it can be observed that the baseline variability within a short-lasting deceleration cannot be consistently or reliably judged on a British CTG (slower recording speed) for "early" as well as "variable" decelerations. This criterion does not add much to the definition but could be a source of unnecessary confusion, introducing variation in interpretation. Thus, the only part of the FIGO definition of "early" decelerations that remains scientifically valid is "coincident in timing with the contraction" with the trough roughly corresponding (not later than $15-20 \mathrm{~s}$ ) to the peak of contraction as described by Hon [21] and consistent with the traditional British practice [14-17].

\section{FIGO (2015) definition of variable deceleration}

(V-shaped) Decelerations that exhibit a rapid drop (onset to 
nadir in less than $30 \mathrm{~s}$ ), good variability within the deceleration, rapid recovery to the baseline, varying size, shape, and relationship to uterine contractions [3].

FIGO (2015) and NICE $(2007,2014)$ have asserted that variable (rapid) decelerations constitute the majority of decelerations during labor $[1,3,30]$ and they translate a baroreceptor-mediated response to increased arterial pressure, as occurs with umbilical cord-compression [3]. The above definition seems precisely the "mishmash" that has confused obstetricians and midwives. Rather than a definition, it seems the opposite - an "abstraction". From the definition, it appears that variable decelerations must have varying (time) relationship to the contractions. Hence, decelerations which consistently occur during the contractions (most common) should not be called "variable". But these get included anyway $[2,13]$ because they are "rapid" and because all decelerations vary in depth and duration anyway! The reference given by FIGO (2015) [3] for this definition is the seminal paper of Ball and Parer [36]. However, this very paper repeatedly states (including in the abstract) that both head-compression and cord-compression cause rapid decelerations with detailed description of evidence [36], thus completely contradicting the FIGO definition [3]. They remarked that head-compression is the likely cause of many variable decelerations classically attributed to cord-compression and these are more common in second stage [36]. Lastly, cord-compression causes FHR drop by a combination of baroreceptor and chemoreceptor mechanisms (hypoxemia) and indeed the latter may be more predominant [12, $33,36]$. The argument that "cord-compression causes rapid decelerations, hence all rapid decelerations are due to cordcompression, hence should be called variable" is one of the two fundamental "formal" fallacies of logic (fallacy by form of the argument alone irrespective of the content) - called "affirming the consequent". Since the criterion of rapid descent or "V" shape does not distinguish between head (early) and cordcompression (variable) decelerations, it should be removed from the definition as it represents a major logical, framing/ anchoring and confirmation fallacy. This "Post-truth" concept should not form part of scientific practice. All types of FHR decelerations necessarily vary in size and to some extent shape (i.e. depth and duration). Thus, variable decelerations should be defined simply based on the variable time relationship to the contractions as described by Hon [21] and consistent with traditional British practice [14-17]. Lastly, abnormal/pathological variable decelerations could be defined as more than $50 \%$ of them having "late components" [2]. Variable decelerations with long duration are likely to have late components or late recovery anyway.

\section{FIGO (2015) definition of late decelerations}

(U-shaped and/or with reduced variability): Decelerations with a gradual onset and/or a gradual return to the baseline and/or reduced variability within the deceleration. Gradual onset and return occurs when more than $30 \mathrm{~s}$ elapses between the beginning/end of a deceleration and its nadir [3].

The use of "and/or" twice in the first sentence allows about nine permutations and combinations. This makes the definition ambiguous, confusing, prone to misinterpretation and clearly wrong. The first "and/or" in the definition conveys a meaning that if there is "gradual return", then "gradual descent" is not a must. The second "and/or" means that provided there is reduced variability within the deceleration, then neither gradual descent nor return is a must (both could be rapid). Perhaps "and/or" seems a mistake. It seems likely that the FIGO expert group intends to mean "plus/minus" in place of "and/or". However, in that case the clause that follows "plus/ minus" could be removed without affecting the meaning of the definition. If the second clause in the definition were to be "plus/minus reduced variability within the deceleration", that clause is superfluous and could be removed without any loss of meaning. Reduced variability within late decelerations does not seem to be a consistent or reliable feature and there is very scant evidence to correlate it with fetal hypoxemia [39] (e.g. Figure 1 shows normal variability within the late deceleration and also more of a "V" than "U" shape). Any purported correlation is by dubious "post-hoc" statistical analysis and in any case very weak and clinically unimportant. Definition of rapid/ gradual descent as "less/more than $30 \mathrm{~s}$ from baseline to nadir" has not been a British practice. Apart being completely arbitrary cut-off, it is associated with irreconcilable contradictions/ aberrations [13, 33]. Moreover, the categorization of FHR decelerations based on this cut-off has been reported to be a major reason for limited usefulness of American three-tier system in clinical practice [6-8]. This unproven and erroneous practice should be critically questioned by the British obstetricians and midwives. The only relevant part of the definition seems to be "late time relationship" to the contraction.

\section{Consequences of Flawed Categorization of FHR Decelerations (NICE, 2007, 2014; FIGO, 2015): Forced Errors}

A consequence of leaving a fundamental error unresolved is likely to be invitation for further errors down the line which can be considered as "forced errors". One example may be the Australia-New Zealand guidelines stating while defining "early" decelerations that they are associated with "reduced baseline variability" (being shallow) and occur between 4 and $7 \mathrm{~cm}$ cervical dilatation [26]. This misconception may be because Chung and Hon in their experiments used ring pessaries of $4-7 \mathrm{~cm}$ size (probably as a matter of convenience) to apply pressure to head to produce early decelerations [37]. Then, having placed vast majority of decelerations into "variable" (cord-compression) category, many experts came up with several injudicious waveform/shape criteria like "truly uniform shape (identical in depth and duration)", symmetrical/asymmetrical shape and most importantly the various waveform criteria (e.g. shoulders) for diagnosing "atypical variable" decelerations $[25-27,30]$. These criteria were challenged $[2,28]$ and recently have been quite rightly dropped $[1,3]$. Following the guidance by NICE (2007) and others [30,25], the commonest cause of pathological CTGs by far was the presence of "atypical variable decelerations" mostly because of lack of a shoulder (small up-shoot). However, concerns were raised [2, 28]. 
Consequently, NICE (2014) [1] made a major change with a somewhat opaque and unreferenced statement, "Do not use the terms "typical" and "atypical" because they can cause confusion". This subcategorization was not only confusing but more importantly unscientific as well [2, 7, 28]. Being required to abandon typical/atypical classification, NICE (2014) replaced it with two thresholds to distinguish abnormal/severe variable decelerations from the benign/less severe ones; namely more than $60 \mathrm{bpm}$ in depth or more than $60 \mathrm{~s}$ duration based on pure consensus but no evidence [1]. This constitutes a leap into the unknown or another trial and error approach. FIGO (2015) on the other hand recommended a very different cut-off of "3 min" duration to classify abnormal variable decelerations again without any evidence [3]. Notwithstanding, a large American expert group recommended two out of three criteria (depth $>60$, dropping below $60 \mathrm{bpm},>60 \mathrm{~s}$ in duration) to be met for pathological variable decelerations, but this seems to miss $70 \%$ of acidemic babies in actual practice conditions $[8,11]$. All of the above cannot be correct at the same time. The FIGO cut-off of 3 min seems suspect and unsafe as discussed later in this paper. NICE seems to be already looking into revising its criteria with consultation just closed. Some centers are devising their own "convenient" modifications of NICE (2014) [1] or FIGO (2015) [3] criteria. The scientific deficit seems obvious. Thus after classifying vast majority of FHR decelerations as "variable" based on substantial framing and confirmation fallacies - a post-truth scenario (see below), the discrimination of their significance remains confused and is left to random or trial criteria of depth/duration. This is also contradicted by the seminal paper of Ball and Parer (1992) [36] referred by FIGO (2015) in their definition [3]. This paper states in the abstract itself is that the depth and duration of "variable decelerations" do not correlate to the reduction in umbilical blood flow (thus possibly the hypoxemia) [36].

Lastly, these post-truth facets of CTG interpretation are about to adversely affect the practice of intermittent auscultation (IA) as discussed in details later.

\section{Occam's Razor}

A well-known long-standing problem-solving principle of "Occam's razor" (law/principle of parsimony) proposes that among competing hypotheses (all things being equal), the one with the fewest assumptions (least complicated) is likely to be true and should be selected [40]. No more assumptions should be made than strictly necessary. Although not an irrefutable principle of logic or science, Occam's razor is a useful and practical guiding principle while formulating concepts. It would have valid application while formulating conceptual frameworks like categorization of FHR decelerations. Hence, it could be argued that proliferation of deceleration waveform descriptions like uniform, symmetrical, U-shaped, V-shaped, gradual/shallow, rapid, variability within decelerations etc. is unnecessary and unhelpful, and should be avoided. These waveform descriptions are subjective, inconsistent, prone to judgement-error, confusing, often wrong, and bear no significant correlation to etiology or fetal hypoxemia. The front-line clinicians as well as experts are prone to confuse these superfluous descriptions [2]. Given the multiple fallacies, contradictions and forced errors associated with the categorization of decelerations based on gradual/rapid descent, it seems high time to revert to the simple "timing-based" traditional British model consistent with that of Hon [14-17, 21].

\section{Rapid Versus Shallow Decelerations: Balance of Arguments}

The arguments in favor of classification based on rapid vs. gradual shape (or $30 \mathrm{~s}$ cut-off for descent time) have been argued to be flawed $[13,33]$. Notwithstanding, the proponents of such a categorization generally claim that the expert consensus must be right (genetic fallacy) or the categorization of decelerations does not really matter after all. Another convenient objection is that any alternative categorization must be shown to improve the detection of fetal hypoxemia by level 1 or 2 evidence. This seems an unjustified position and misapplication of principles of EBM especially when NICE (2014) states that evidence for significance of most FHR parameters is of poor quality [1]. But this seems more of a failure of the application of EBM rather than failure of CTG interpretation or EBM itself. The proponents should be able to defend the validity of their case by rational arguments and scientific observations. Their case should withstand a 2,500-year-old Socratic method of analysis. Let's hope Socrates did not waste his "death". It is not an uncommon mistake to confuse EBM or lack of it almost as a "displacement activity" to disqualify critical observation and reasoning. Another argument offered is that as long as clinicians follow some authoritative guidelines, then they will be protected professionally and medico-legally. However, the primary and principal purpose of clinical guidelines is to protect/benefit patients and then secondarily clinicians. A possible criticism of this paper would be that while the guidelines describe many other aspects of CTG like FHR baseline, variability, accelerations, clinical situations and decision making, this paper only addresses FHR decelerations in isolation. Most guidelines repeat similar description of other aspects of CTG interpretation which are relatively non-controversial. On the other hand, FHR decelerations represent a major current controversy and also remain center-stage (most important) in CTG interpretation [12], and hence are the specific focus of this paper. Leading experts from New Zealand and America (including Prof. J. Parer) in a seminal paper gave an illustration of CTG (Fig. $5[3,12])$ in a case of known cord prolapse (compression) specifically to demonstrate "variable decelerations" [12]. This is a fairly typical CTG observed in cases of cord prolapse or severe hypoxemia from other causes. All deep decelerations will look rapid on CTG with recording speed of $1 \mathrm{~cm} / \mathrm{min}$ (Fig. 5). In this illustration, the slope of FHR drop slows down after the peak of contraction but the zenith is reached much later during the relaxation phase (descent time well above 30 s) and recovery is complete well after the contractions. Thus, based on FIGO and ACOG definitions [3-5], these decelerations due to confirmed cord-compression would be consid- 


\section{Cardiotocogram recording of FHR and contractions from a term human fetus who had a cord prolapse in early labour}
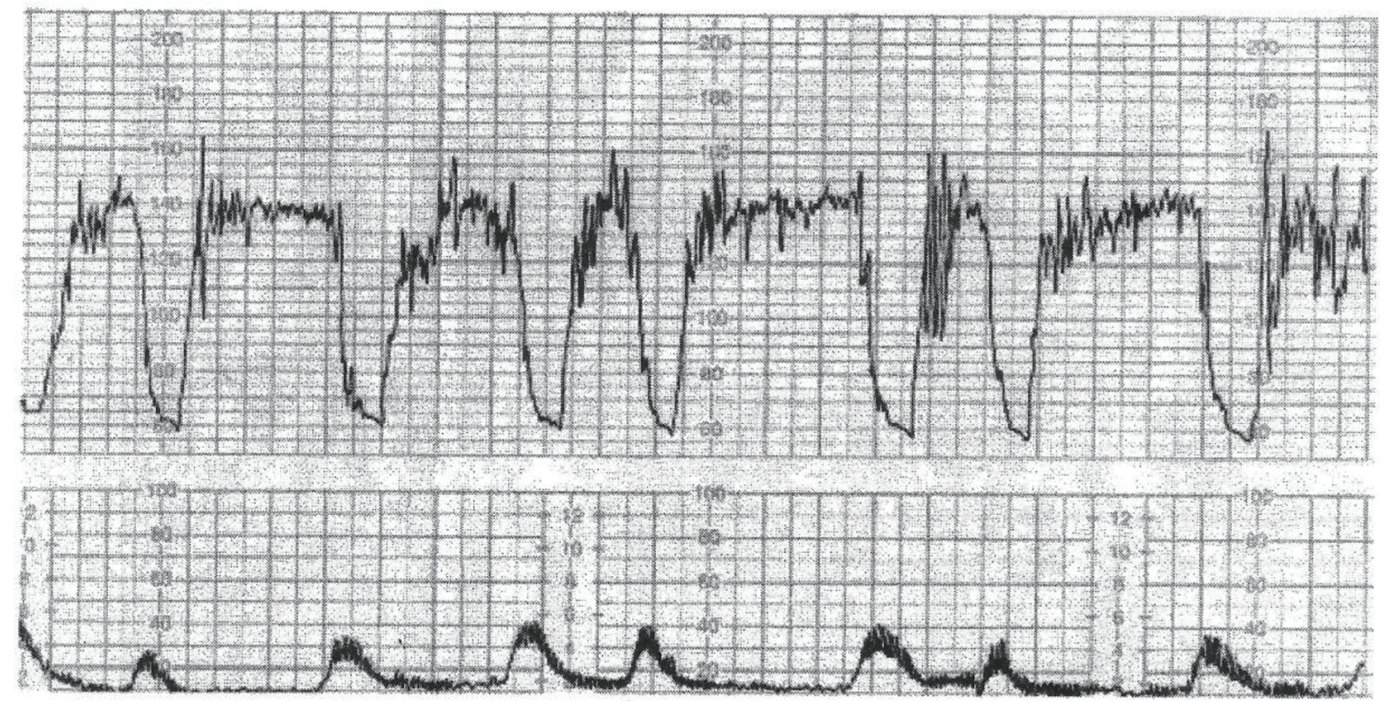

Figure 5. CTG in a case of cord prolapse showing decelerations classed as "variable" by experts [12]. Reproduced with thanks from Westgate et al, Am J Obstet Gynecol. 2007;197:236.e1-11 [12]. Although these decelerations "look" rapid (paper speed 1 $\mathrm{cm} / \mathrm{min}$ ), the "descent-time" is well over $30 \mathrm{~s}$. Moreover, these proven cord-compression decelerations look very different from the vast majority of decelerations coincident with contractions. By FIGO (2015) guidelines these variable decelerations could be mistakenly classed as benign as they are less than three minutes in duration [3].

ered to have slow descent (gradual) and classed as "late" and not "variable". This example confirms that the "descent-time" does not reliably correlate with etiology and cord-compression may often cause late decelerations [12]. However, the front-line clinicians and even experts are very likely to call these decelerations "variable" because of marked "rapid" appearance and if one applies the FIGO (2015) [3] cut-off of "3-min duration", then these cord-prolapse decelerations would be classed as benign (well below $3 \mathrm{~m}$ duration). Thus this expert illustration itself exemplifies the risks of fallacious "rapid/gradual" criteria and arbitrary cut-offs of duration in the guidelines. Is it possible to call these decelerations (Fig. 5) "variable" because the onset is early in the contraction but nadir and recovery are late? But this proves that the "variable time relationship" would be the defining feature and descent time $(<30 \mathrm{~s})$ is certainly not. Moreover, the duration of these decelerations is about 2 min which would prove that the FIGO (2015) criterion of 3 min duration for abnormal variable decelerations [3] is clearly wrong and may pose a risk to patients. On the balance it may be better to call these decelerations "late" because of very consistent late nadir and late recovery during every contraction. The term "variable" may be best reserved for the decelerations that vary in timing in relation to different contractions. Cord-compression could be comparable to holding one's breath and fetal hypoxemia ensues fairly quickly because of compression of umbilical vein which brings oxygenated blood to the fetus. This triggers a drop in FHR due to chemoreceptor mechanism (in addition to baro-reflex). Umbilical venous compression would be relieved not at the height of contraction but indeed much later during the relaxation phase (Fig. 3A and B). Hence, the (most common) decelerations with rapid descent and trough corresponding to peak of contraction cannot be scientifically explained by cord-compression but this is in fact contradicted by pathophysiological hypotheses as well as animal studies as described in details elsewhere [33].

\section{Evidence From Doppler Studies During Labor and Placental Compression Hypothesis}

Several studies of umbilical artery blood flow velocity waveform (UABVW) using Doppler during FHR deceleration in labor have been performed since late 1980s but very little definitive or clinically usable information is available [41]. These studies generally demonstrate a reduction in umbilical arterial blood flow and increase in systolic/diastolic (S/D) ratio during decelerations $[36,41,42]$ but this cannot be presumed to confirm cord-compression. The UABVW is influenced by many factors including downflow resistance, cardiac output and heart rate [36, 42]. Fairlie et al (1989) commented that the abnormal waveform patterns associated with FHR decelerations could be due to prolongation of diastole resulting in apparent loss of end-diastolic frequencies [42]. Somerset et al (1993) showed that the UABVW analysis (raised S/D ratio) failed to correlate with fetal distress due to cord-complications [43] and quoted that Malcus et al (1991) had actually shown a negative correlation between the two [44]. The observation that many rapid decelerations coincident with contractions are ameliorated by saline amnioinfusion was thought to confirm cord-compression as the underlying etiology. But the largest and the only trial with robust methodology showed 
that amnioinfusion did not reduce the incidence of fetal hypoxemia/acidemia [1]. Hence alternative explanations should be considered. The placenta, a large soft organ, is very likely to be compressed during contractions rather than or more often than the cord $[2,13]$. This would increase the resistance in placental fetal circulation (downflow resistance) explaining the reduction in umbilical arterial blood flow and increase in $\mathrm{S} / \mathrm{D}$ ratio. But this is unlikely to result in fetal hypoxemia as more oxygenated blood will be passed to the fetus through the umbilical vein during the contraction (placental compression phase). At the same time, this would cause a drop in FHR (early deceleration) via baroreceptor mechanism due to increase in fetal blood volume/pressure. The placental compression and thus the vascular resistance (afterload) would start dropping immediately after the peak of contraction with reversal of net blood flow, i.e. more blood will flow from fetus to the placenta. This would lead to recovery of FHR deceleration by baro-receptor mechanism with nadir corresponding to peak of contraction $[2,13]$. This seems an attractive auxiliary hypothesis to explain early decelerations in addition to the primary hypothesis of head-compression $[13,33]$. It also explains why many rapid decelerations coincident with contractions are ameliorated with saline amnioinfusion without improving hypoxemia. The placental compression hypothesis raises an interesting possibility that it could serve as a partcompensatory mechanism in that oxygenated blood is pushed to the fetus partly offsetting the significant drop in maternal uteroplacental perfusion during the contraction phase (note negative correlation between UABVW changes and fetal distress [44]). However, apart from adding weight to the concept that FHR decelerations coincident with contractions are benign (early), the placental compression hypothesis may not necessarily be of any other clinical relevance in the present state of knowledge. Hon made a very brief mention of "placental compression" when he gave an illustration of early decelerations due to head-compression on a CTG in twin labor [45]. Hon showed that the presenting twin whose vertex was dilating cervix consistently had decelerations with contractions while the second twin with head out of pelvis had no decelerations [45]. He commented that both twins were subjected to similar "compression of placenta" (both amniotic sacs intact) during contractions, and hence this could not have been the cause of decelerations observed. Interestingly, a careful examination of the FHR record provided by Hon [45] reveals that the "descent-time" of these decelerations most likely due to head-compression is about $10-15 \mathrm{~s}$ (rapid rather than gradual) contrary to FIGO and ACOG definitions [3-5].

\section{Pathophysiology of Decelerations: Hypoxic Vs. Non-Hypoxic Vagal Reflex}

Currently, the vast majority of decelerations are (mistakenly) postulated to be due to cord-compression and then the effort gets mislaid on discriminating which sorts of cord-compression decelerations are likely to lead to fetal hypoxemia/acidemia. This has not been successful so far $[6-8,11]$, because it seems a wrong path to proceed. There is no evidence that repeated cord-compression during contractions is the cause of significant number of cases fetal acidemia in human labor. On the other hand, the most common cause of fetal hypoxemia/ acidemia is contraction-induced reduction in maternal uteroplacental perfusion. That is why there is more incidence of fetal hypoxemia in hypertonic uterine action, intrauterine growth retardation (IUGR), preeclampsia and other cases where uteroplacental perfusion is already compromised. With the focus on fetal hypoxemia, FHR decelerations can be said to be of two main types, one due to benign (non-hypoxemic) parasympathetic (vagal) reflex and the other due to hypoxic (chemoreceptor) vagal reflex \pm direct suppression of myocardium in later stages $[2,17,27]$. The clue to differentiating this is in the "timing" rather than "rapid/gradual shape", since hypoxemia during contraction has a lag time to develop or worsen $[2,17$, 27]. FHR decelerations which start recovering immediately at or after the peak of contraction (early timing) do not have this hypoxemic component and hence it would be important to appropriately recognize them as benign ("early") which was achieved with the previous traditional British categorization [2, 14-17]. All experts emphasize the importance to understand the underlying pathophysiology [12] but the primary focus on rate of descent by the ACOG and FIGO classifications [3-5] seems a hazardous distraction and contradiction of these most important pathophysiological principles [13].

\section{Myths, Post-truth Phenomenon and Backfire Ef- fect}

"Backfire effect" describes a common phenomenon that when the deepest convictions are challenged by contradictory evidence, false beliefs entrench even deeper and facts do not seem to matter. Resistance by American obstetricians to change is understandable as they have not known any other system for a few decades. However, for the British obstetricians and midwives, the proof of the pudding is in eating. They have practiced a purely timing-based categorization of decelerations from 1970s to 2007 and found it generally satisfactory (if not perfect) $[2,24]$. It was found certainly more useful than the current categorization based on rapid/gradual descent which has found wanting in recent studies [7, 11]. Major framing and confirmation fallacies are incompatible with scientific practice and need to be rectified as we will be accountable to the larger scientific community and our patients. Knowingly teaching false pathophysiology/nomenclature is not science but a "posttruth phenomenon" (claims asserted as correct even if they have no actual basis in fact and are what people want to believe because they fit beliefs they already have). Following common myths need to be rejected: 1) Head-compressions cause gradual (U-shaped) decelerations; 2) Vast majority of decelerations are "variable" with cord-compression being the main causation; 3) What label we attach to FHR decelerations does not matter as long as everyone follows the same convention. In fact, categorization of decelerations is the framework by which we learn, recognize, analyze and interpret the most important and common complex patterns on CTG. Getting "boxed in" by wrong concepts is hazardous for pattern recognition. 
Table 1. Proposed (British) Definitions of FHR Decelerations

\begin{tabular}{ll}
\hline Early decelerations & $\begin{array}{l}\text { Recurrent/repetitive slowing of FHR (depth }>15 \text { beats/min) with onset early in the contraction and return to baseline } \\
\text { at the end of contraction. }\end{array}$ \\
Late decelerations & $\begin{array}{l}\text { Recurrent/repetitive slowing of FHR with onset mid to end of contraction and nadir more than } 20 \mathrm{~s} \text { after peak of } \\
\text { contraction and ending after the contraction. If baseline variability is less than } 5 \text { beats/min, then the definition would } \\
\text { include decelerations less than } 15 \text { beats/min. }\end{array}$ \\
Variable decelerations & $\begin{array}{l}\text { Recurrent/repetitive slowing of FHR with varying time relationship to the contraction cycle. They tend to markedly } \\
\text { vary in shape. (Variable decelerations could be further classed as abnormal or complicated (the term atypical is now } \\
\text { tainted) if more than } 50 \% \text { of them have late components, but this has to be interpreted in the clinical context.) }\end{array}$ \\
\hline
\end{tabular}

These definitions are very similar to the traditional British practice between 1970 and 2007 [14-17] and of Edward Hon [21].

\section{Safeguard Intermittent Auscultation (IA)}

Another forced error in the FIGO (2015) IA auscultation guidelines is to recommend FHR auscultation "during contractions" while performing IA in low risk labors and advice to commence CTG if decelerations are detected coincident with contractions [46]. This is a direct consequence of the flawed categorization of the vast majority of decelerations coincident with contractions as "variable" requiring further elucidation. However, this recommendation would be against the long and well-established British obstetric and midwifery practice of disregarding FHR decelerations limited to contractions (early) because they are essentially benign $[1,25,29,30]$ based on long experience as well as the pathophysiological basis. Some experts forward an opinion that decelerations confined to contractions could be pathological but without any good quality scientific proof whatsoever. Moreover, let us suppose CTG is commenced when FHR decelerations are suspected/detected during contractions. On CTG, these will be called "variable decelerations" (rapid descent) and then classed abnormal only if they last longer than 3 min according to FIGO (2015) CTG guidelines [3]. It is obvious that decelerations of duration $>$ 3 min would extend well beyond the end of contraction and would be detected even if the auscultation was not performed during the contraction. Thus, FIGO guidelines seem to contradict themselves [3, 46]. British midwifery organizations should recommend to avoid auscultation during contractions or, if performed, to disregard any decelerations coincident with contractions. Otherwise, it is likely that a large number of cases would be unnecessarily switched over to CTG or transferred to hospital obstetric units, with resultant disempowerment of patients and midwifery practice and increased medical intervention [47]. The benefits of CTG over IA have been seriously questioned [48] and misconceived switch-over of IA to CTG should be resisted [47]. However, judicious and more extended auscultation (but disregarding any decelerations confined to contractions) is important to establish baseline FHR [49].

\section{Conclusions}

Errors in the intrapartum CTG interpretation are high on the agenda of RCOG with its "every baby counts" initiative. Dysfunctional categorization of FHR decelerations has led to false alarm fatigue and confusion with consequent loss of confidence and demoralization of midwives and junior obstetricians $[2,28]$. The unscientific criteria of atypical variable decelerations have now been abandoned. However, in many British hospitals, the junior obstetricians and midwives have stopped diagnosing any early and late decelerations at all in the last few years. This may have compromised patient safety. Unfortunately, the opportunity to reform the basic fundamental categorization of early/late/variable decelerations seems to have been missed by NICE (2014) and other guidelines [1$3]$. Many British obstetric units are debating whether to adopt NICE (2014) or FIGO (2015) guidelines [1, 3] or other local modifications. The FIGO, NICE and ACOG categorization of FHR decelerations based on rapid vs. shallow/gradual decelerations [1, 3-5] seem purely ideological and do not reflect the underlying etiology, pathophysiology or fetal condition [13]. They also seem to embody contradictions and major framing/ anchoring and confirmation fallacies and constitute "posttruth" scenario. It is best not to base the categorization of decelerations on presumptive etiology or shape of deceleration waveform. The current categorization of decelerations [1, 3-5] is very different from and contradictory to the one proposed by Hon [21], and hence should not borrow the terminology "early/late/variable" and continue to claim legitimacy from it. To be open and transparent to the clinicians, the current categorization is best designated as "rapid" and "gradual" decelerations, with gradual being further divided into "gradual early" (not found) and "gradual late" [33]. NICE has not provided specific definitions of different types of FHR decelerations leaving a critically important void to be filled $[1,30]$. The obstetricians and midwives and NICE should debate and adopt the traditional British definitions of FHR decelerations based solely on time relationship to the contractions as manifest in the nomenclature "early/late/variable" itself (Table 1) [13-17, 21]. These are more scientific and reflect the pathophysiological basis of hypoxic vs. non-hypoxic decelerations [2, 13, 21$]$. This physiological classification of types of FHR decelerations can be practiced with most three-tier systems similar to British one provided the smaller proportion of variable decelerations are classed as abnormal based on presence of repetitive late components. The confidence and reassurance for the birth attendants and patient safety will be contingent on the ability to confirm normality by categorizing common benign (early) decelerations correctly and then being able to focus/act on the smaller number of pathological ones [2]. This will also facilitate future developments and research. 


\section{Acknowledgments}

The author greatly appreciates the contribution by the researchers in the field of intrapartum monitoring. He is indebted to late Prof. Parer for his excellent work in this field. He is thankful to his Institute's Library staff for excellent and timely help.

\section{Conflicts of Interest}

The author has no conflicts of interest or funding to declare. Most of the concepts presented have been previously communicated to relevant guideline groups. These represent opinion only and do not necessarily reflect practice. This review includes substantial new additional information and concepts in addition to previous publications to assist the debate.

\section{References}

1. National Institute for Health and Clinical Excellence (2014). National Collaborating Centre for Women's and Children's Health. Intrapartum care - Care of healthy women and their babies during childbirth. Guideline No 190., London (UK). [Accessed 15 March 2016]. Available from URL: http://www.nice.org.uk/guidance/cg190/ resources/guidance-intrapartum-care-care-of-healthywomen-and-their-babies-during-childbirth-pdf.

2. Sholapurkar SL. Interpretation of British experts' illustrations of fetal heart rate (FHR) decelerations by Consultant Obstetricians, Registrars and midwives: A prospective observational study-Reasons for major disagreement and implications for clinical practice. Open J Obstet Gynecol. 2013;3(3):454-465.

3. Ayres-de-Campos D, Spong CY, Chandraharan E. FIGO consensus guidelines on intrapartum fetal monitoring: Cardiotocography. Int J Gynaecol Obstet. 2015;131(1):1324.

4. ACOG Practice Bulletin No. 106: Intrapartum fetal heart rate monitoring: nomenclature, interpretation, and general management principles. Obstet Gynecol. 2009;114(1):192-202.

5. Electronic fetal heart rate monitoring: research guidelines for interpretation. National Institute of Child Health and Human Development Research Planning Workshop. Am J Obstet Gynecol. 1997;177(6):1385-1390.

6. Coletta J, Murphy E, Rubeo Z, Gyamfi-Bannerman C. The 5-tier system of assessing fetal heart rate tracings is superior to the 3-tier system in identifying fetal acidemia. Am J Obstet Gynecol. 2012;206(3):226 e221-225.

7. Cahill AG, Roehl KA, Odibo AO, Macones GA. Association and prediction of neonatal acidemia. Am J Obstet Gynecol. 2012;207(3):206 e201-208.

8. Clark SL, Nageotte MP, Garite TJ, Freeman RK, Miller DA, Simpson KR, Belfort MA, et al. Intrapartum management of category II fetal heart rate tracings: towards standardization of care. Am J Obstet Gynecol. 2013;209(2):89-97.
9. Sartwelle TP, Johnston JC. Cerebral palsy litigation: change course or abandon ship. J Child Neurol. 2015;30(7):828-841.

10. INFANT Study. A study of decision support in women requiring CTG monitoring in labour. Clinical Trials Unit - University College London - Gower Street - London. http://www.ucl.ac.uk/cctu/researchareas/womenshealth/ infant.

11. Clark SL, Hamilton EF, Garite TJ, Timmins A, Warrick PA, Smith S. The limits of electronic fetal heart rate monitoring in the prevention of neonatal metabolic acidemia. Am J Obstet Gynecol. 2017;216(2):163 e161-163 e166.

12. Westgate JA, Wibbens B, Bennet L, Wassink G, Parer JT, Gunn AJ. The intrapartum deceleration in center stage: a physiologic approach to the interpretation of fetal heart rate changes in labor. Am J Obstet Gynecol. 2007;197(3):236 e231-211.

13. Sholapurkar SL. Categorization of Fetal Heart Rate Decelerations in American and European Practice: Importance and Imperative of Avoiding Framing and Confirmation Biases. J Clin Med Res. 2015;7(9):672-680.

14. Williams J, Blanchard J. Suspicious and Pathological Fetal Heart Traces. In: Electronic monitoring of fetal heart. 1st edition. Hale, Cheshire, UK; Books for midwives Press. 1996; p. 24-42.

15. Ritchie JWK. Fetal surveillance. In: Whitfield CR, editors, Dewhurst's Textbook of Obstetrics and Gynaecology for Postgraduates, 4th edition. Oxford, UK: Blackwell Scientific Publications. 1986; p. 442-462.

16. Malvern J. The clinical management of labour. In: Turnbull A, Chamberlain G, editors. Obstetrics, 1st edition. London, UK: Churchill Livingstone. 1989; p. 713-723.

17. Hibbard BM. Assessment of fetal condition during labour. In: Hibbard BM, editor. Principles of Obstetrics, 1st edition. London, UK: Butterworth \& Co. (Publishers) Ltd. 1988; p. 472-489.

18. Cognition: How Your Mind Can Amaze and Betray You - Crash Course Psychology \#15. https://www.youtube. com/watch?v=R-sVnmmw6WY.

19. Nickerson RS. Confirmation bias: a ubiquitous phenomenon in many guises. Rev Gen Psychol. 1998;2(2):175200.

20. Pines JM. Profiles in patient safety: confirmation bias in emergency medicine. Acad Emerg Med. 2006;13(1):9094.

21. Hon EH, Quilligan EJ. Electronic evaluation of fetal heart rate. IX. Further observations on "pathologic" fetal bradycardia. Clin Obstet Gynecol. 1968;11(1):145-167.

22. Mendez-Bauer C, Poseiro JJ, Arellano-Hernandez G, Zambrana MA, Caldeyro-Barcia R. Effects of atropine on the heart rate of the human fetus during labor. Am J Obstet Gynecol. 1963;85:1033-1053.

23. Greenhalgh T, Howick J, Maskrey N. Evidence based medicine: a movement in crisis? BMJ. 2014;348:g3725.

24. Sholapurkar SL. The conundrum of vanishing early decelerations in British obstetrics, a step backwards? Detailed appraisal of British and American classifications of fetal heart rate decelerations - fallacies of emphasis on waveform and putative aetiology. J Obstet Gynaecol. 
2012;32(6):505-511.

25. eFM. Fetal Heart Rate Monitoring. An e-learning resource improving the interpretation of EFM in labour and its subsequent management. Department of Health Programme in partnership with Royal College of Obstetricians and Gynaecologists and the Royal College of Midwives, United Kingdom, 2011. [Accessed April 20, 2014].

26. OFSEP. Online Fetal Surveillance Education Programme. The Royal Australian and New Zealand College of Obstetricians and Gynaecologists. June 2011. Available from URL: www.ranzcog.edu.au/publication/womens-healthpublications/intrapartum-fetal-surveillance-clinicalguidelines.html [Accessed 1st March 2015.].

27. K2 Fetal Monitoring Training System. K2 Medical Systems, Plymouth, United Kingdom; 2009. Available from URL: http://www.k2ms-university.com [Accessed 5 January 2014].

28. Sholapurkar SL. Are 'early' and 'late' fetal heart rate decelerations extinct? A survey of British midwives and analysis of controversies, facts and fiction. British Journal of Midwifery. 2013;21(12):710-715.

29. Royal College of Obstetricians and Gynaecologists. Clinical Effectiveness Support Unit. The use of electronic fetal monitoring. The use and interpretation of cardiotocography in intrapartum fetal surveillance. Evidence based Clinical Guideline No 8, London, RCOG Press, UK, 2001.

30. National Collaborating Centre for Women's and Children's Health. Intrpartum Care - Care of healthy women and their babies during childbirth. National Institute for Health and Clinical Excellence (NICE) clinical guideline 55, London, UK, 2007. Available from URL: http://guidance.nice.org.uk/CG55, [Accessed 15 October 2012].

31. Lee CY, Di Loreto PC, O'Lane JM. A study of fetal heart rate acceleration patterns. Obstet Gynecol. 1975;45(2):142-146.

32. Gibb D, Arulkumaran S. Control of fetal heart rate and NICE guidelines. In: Gibb D, Arulkumaran S, editors. Fetal Monitoring in Practice, 3rd edition. London, UK: Churchill Livingstone. 2007; p. 27-44.

33. Sholapurkar SL. Critical evaluation of American categorization of fetal heart rate (FHR) decelerations and three tier classification-Shortcomings, contradictions, remedies and need for debate. Open Journal of Obstetrics and Gynecology. 2013;3(3):362-370.

34. Garite TJ. Intrapartum fetal evaluation. In: Gabbe SG, Niebyl JR, Simpson JL, editors. Obstetrics: Normal and problem pregnancies, 4th edition. New York, USA, Churchill Livingstone. 2002; p. 395-429.
35. Court DJ, Parer JT. Experimental studies of fetal asphyxia and fetal heart rate interpretation. In: Nathanielsz PW, Parer JT, editors. Research in Perinatal Medicine (I). New York, USA: Perinatalogy Press. 1984; p. 113-169.

36. Ball RH, Parer JT. The physiologic mechanisms of variable decelerations. Am J Obstet Gynecol. 1992;166(6 Pt 1):1683-1688; discussion 1688-1689.

37. Chung F, Hon EH. The electronic evaluation of fetal heart rate. I. With pressure on the fetal skull. Obstet Gynecol. 1959;13(6):633-640.

38. Rech W. Untersuchungen uber die Herztatigkeit des Fetus. III. Die Wirkung des Kopfdruckes auf die Frequenz des fetalen Herzchlages. Arch Gynaecol, 1933;154:4757.

39. Cahill AG, Roehl KA, Odibo AO, Macones GA. Association of atypical decelerations with acidemia. Obstet Gynecol. 2012;120(6):1387-1393.

40. Occam's razor. Wikipedia. Available from URL: https:// simple.wikipedia.org/wiki/Occam\%27s_razor.

41. Mihu D, Diculescu D, Costin N, Mihu CM, Blaga L, Ciortea R, Malutan A. Applications of Doppler ultrasound during labor. Med Ultrason. 2011;13(2):141-149.

42. Fairlie FM, Lang GD, Sheldon CD. Umbilical artery flow velocity waveforms in labour. Br J Obstet Gynaecol. 1989;96(2):151-157.

43. Somerset DA, Murrills AJ, Wheeler T. Screening for fetal distress in labour using the umbilical artery blood velocity waveform. Br J Obstet Gynaecol. 1993;100(1):55-59.

44. Malcus P, Gudmundsson S, Marsal K, Kwok HH, Vengadasalam D, Ratnam SS. Umbilical artery Doppler velocimetry as a labor admission test. Obstet Gynecol. 1991;77(1):10-16.

45. Hon EH. The electronic evaluation of the fetal heart rate; preliminary report. Am J Obstet Gynecol. 1958;75(6):1215-1230.

46. Lewis D, Downe S. FIGO consensus guidelines on intrapartum fetal monitoring: Intermittent auscultation. Int $\mathbf{J}$ Gynaecol Obstet. 2015;131(1):9-12.

47. Sholapurkar SL. Amendments in electronic fetal monitoring and intermittent auscultation. British Journal of Midwifery. 2016;24(9):665-667.

48. Nelson KB, Sartwelle TP, Rouse DJ. Electronic fetal monitoring, cerebral palsy, and caesarean section: assumptions versus evidence. BMJ. 2016;355:i6405.

49. Sholapurkar SL. Intermittent Auscultation in Labor: Could It Be Missing Many Pathological (Late) Fetal Heart Rate Decelerations? Analytical Review and Rationale for Improvement Supported by Clinical Cases. J Clin Med Res. 2015;7(12):919-925. 